\title{
Rarely pure and never simple: interpreting investigation results in the clinical context
}

\section{DOI: $10.7861 /$ clinmed.ed.20.6.1}

Myocardial infarction is one component of the spectrum of acute coronary syndrome, ranging from acute myocardial ischaemia to unstable angina and non-ST segment elevation myocardial infarction. Troponin testing is a core part of the algorithm for the diagnosis of myocardial infarction in the emergency department. In this edition of Clinical Medicine we are pleased to publish a paper by Hinton et al looking at the clinical implications of troponin concentrations at the upper limit of normal. ${ }^{1}$ Building on their previously published CHARIOT study, which studied the normal range of troponin for hospital inpatients, the authors have focused on purely inpatient outcomes in a consecutive series of over 5,000 patients presenting to the emergency department. ${ }^{2}$ They show that troponin concentrations were associated with hospital admission, length of stay and mortality, and raises the question of whether troponin may represent a biomarker for non-cardiac causes of hospital attendance. Publishing such clinically relevant material from post-hoc analysis of prospectively collected data is a key part of the scope of Clinical Medicine and we look forward to other similar submissions.

Chronic diarrhoea affects $5 \%$ of the population, and pancreatic exocrine insufficiency is a not infrequent cause. Diagnostic testing using faecal elastase-1 in stool often generates equivocal results, and Shandro and colleagues present a paper on the factors predicting a positive result in cases where there is such diagnostic doubt. ${ }^{3}$ They identify that up to half of such repeat tests have a normal result on re-testing. The commonest cause of such insufficiency is chronic pancreatitis, an entity arising from progressive inflammation and fibrosis of the pancreas which results in the syndrome of abdominal pain, endocrine and exocrine insufficiency. The paper highlights the importance of assessing these common problems (chronic diarrhoea and abdominal pain) in the broad clinical frame of history, radiological and serological features, not just relying on a single stool test.

Another related paper on the interpretation of first-line investigation in common conditions comes from Nepal et al who report a quality improvement project on the diagnostic accuracy of point-of-care ultrasound versus departmental ultrasound in patients with acute kidney injury. ${ }^{4}$ While providing data on the reliability to diagnose renal tract obstruction in this setting, the authors also point to the importance of departmental scanning being considered in patients presenting atypically.

A strong feature of our online content in this edition are papers reflecting the current clinical phase of the COVID-19 pandemic. There are papers on patient self-stratification methodology to identify those in need of shielding, the clinical sensitivity of a single polymerase chain reaction test for diagnosing COVID-19 using convalescent antibody as a comparator, the case-mix in medical take, use of a telecommunications approach to reduce face-to-face exposure time during inpatient care of patients and medical training. ${ }^{5-10}$ This latter topic, of the impact of the pandemic on training, was the subject of the inaugural Twitter RCP Journal Club (\#RCPjournalclub), hosted live on 05 November 2020.

A particularly important paper by Kerr and Stacpoole relates to the presentation of COVID-19 in the elderly. ${ }^{11}$ This is especially relevant as we move to optimal use of case identification and contact tracing. The report also describes the epidemiology of infection in a cohort late into lockdown, identifying that cases presenting to hospital predominantly originate from those in contact with health and care systems, as opposed to being purely community-acquired.

The pandemic continues to educate and challenge our understanding of disease and health care provision, and we are delighted to continue to receive high-quality manuscripts which help us understand these a little more clearly.

Anton Emmanuel Editor-in-chief

\section{References}

1 Hinton J, Mariathas M, Gabara L et al. Distribution of contemporary sensitivity troponin in the emergency department and relationship to 30-day mortality: The CHARIOT-ED substudy. Clin Med 2020;20:528-34.

2 Mariathas M, Allan R, Ramamoorthy S et al. True 99th centile of high sensitivity cardiac troponin for hospital patients: prospective, observational cohort study. BMJ 2019;364:1729.

3 Shandro BM, Ritehnia J, Chen J, Nagarajah R, Poullis A. The investigation and management of pancreatic exocrine insufficiency: $A$ retrospective cohort study. Clin Med 2020;20:535-40.

4 Nepal S, Dachsel M, Smallwood N. Point-of-care ultrasound rapidly and reliably diagnoses renal tract obstruction in patients admitted with acute kidney injury. Clin Med 2020;20:541-4.

5 Reilly E, Skeoch S, Hardcastle S et al. Evaluation of a patient selfstratification methodology to identify those in need of shielding during COVID-19. Clin Med 2020;20:e212-4.

6 Holborrow A, Asad H, Porter L et al. The clinical sensitivity of a single SARS-CoV-2 upper respiratory tract PCR test for diagnosing COVID-19 using convalescent antibody as a comparator. Clin Med 2020;20:e209-11.

7 Ho KMA, Anandhakrishnan A, Mahay A. How COVID-19 has changed the unselected medical take: an observational study. Clin Med 2020; 20:e229-33.

8 Tomlinson J, Khan S, Page G. Incorporating SPACES recommendations to the COVID-19 ward care approach at the Royal Bournemouth Hospital. Clin Med 2020;20:e234-7.

9 Sasitharan A. COVID-19: The impacts on foundation training. Clin Med 2020;20:e253-4.

10 Phillips G, Jones M, Dagg K. Restarting training and examinations in the era of COVID-19: A perspective from the Federation of Royal Colleges of Physicians UK. Clin Med 2020;20:e248-52.

11 Kerr AD, Stacpoole SRL. Coronavirus in the elderly: a late lockdown UK cohort. Clin Med 2020;20:e222-8.

\begin{tabular}{|c|c|c|c|c|}
\hline \multicolumn{5}{|c|}{ Members of the editorial board } \\
\hline Cono Ariti & Albert Edwards & Alexandra Lake & Gerrard Phillips & Trainee associate editors: \\
\hline Paul Belcher & Anton Emmanuel & Yash Mahida & Sushma Saksena & Tessa Cacciottolo \\
\hline Rodger Charlton & Kate Evans & Nick Manning-Cork & Philip Smith & Rajan Pooni \\
\hline Tim Chevassut & Laura Ferrigan & Chris Marguerie & Angela Star & Anenta Ramakrishnan \\
\hline Tahseen Chowdhury & Tevfik Ismail & Martin McKee & Cameron Swift & \\
\hline Richard Corbett & Vikas Kapil & Andrew Medford & Rhys Thomas & \\
\hline Elaine Dennison & Neelam Kumar & Mehool Patel & & \\
\hline
\end{tabular}

